\title{
Canada's Pandemic Influenza Preparedness: Surveillance strategy
}

\author{
B Henry ${ }^{1,2}$ on behalf of the Canadian Pandemic Influenza Preparedness (CPIP) Task Group*
}

\section{Abstract}

The Canadian Pandemic Influenza Preparedness: Planning Guidance for the Health Sector (CPIP) is a guidance document that outlines key health sector preparedness activities designed to ensure Canada is ready to respond to the next influenza pandemic. This article outlines Canada's pandemic influenza surveillance strategy as described in the CPIP Surveillance Annex. The strategy builds on the surveillance activities used for seasonal influenza and incorporates lessons learned from the $2009 \mathrm{H} 1 \mathrm{~N} 1$ pandemic, including improved information sharing, improved electronic links among Federal/Provinical/Territorial (FPT) partners and improved surveillance for Indigenous communities. Key elements of the surveillance strategy include early detection and investigation of a novel influenza virus through the reporting of cases or clusters of severe acute respiratory infections and laboratory detections of novel influenza viruses. Community-based surveillance will provide information on clinical severity, age groups affected and risk factors associated with severe disease. Severe outcome surveillance will capture data on hospitalizations and deaths. Laboratory surveillance will include weekly reports of respiratory virus detections. The response activities are adaptable to the demands of different levels of pandemic activity and impact, supported by a set of triggers for the activation and deactivation. Surveillance will be linked with other response components, such as communications, research, assessment and evaluation. This is an evergreen document that will be updated regularly.

\section{Affiliations}

${ }^{1}$ CPIP Task Group Chair

${ }^{2}$ Office of the Provincial Health Officer, Victoria, BC

*Correspondence: CPIPTGSecretariatGTPCPSecretariat@phac-aspc. gc.ca

Suggested citation: Henry B on the behalf of the Canadian Pandemic Influenza Preparedness (CPIP) Task Group. Canada's Pandemic Influenza Preparedness: Surveillance strategy. Can Commun Dis Rep. 2018;44(1):14-7.

https://doi.org/10.14745/ccdr.v44i01a04

\section{Introduction}

Public health surveillance, the systematic collection and analysis of health data needed for planning, implementing and evaluating public health measures, is a key function in an influenza pandemic (1). Timely surveillance data provide information on the impact of the novel virus and the spread of the pandemic through different regions and populations, informing decisions on pandemic control elements such as the use of vaccines and other interventions.

Canada's pandemic surveillance strategy, described in the Surveillance Annex (2) to the broader Canadian Pandemic Influenza Preparedness: Planning Guidance for the Health Sector (CPIP) (3), provides technical advice and operational guidance for federal, provincial and territorial (FPT) ministries of health and other participants in surveillance activities, such as health professionals and laboratories. It describes surveillance activities that are carried out collaboratively by all FPT jurisdictions, coordinated at the national level by the Public Health Agency of Canada (PHAC). The response activities are adaptable to the demands of different levels of pandemic activity and impact, supported by a set of triggers for the activation and deactivation of specific surveillance activities at different stages of a pandemic.

The surveillance strategy incorporates a number of lessons learned about the surveillance function in the $2009 \mathrm{H} 1 \mathrm{~N} 1$ influenza pandemic. These lessons include improved information sharing among federal and provincial and territorial (PT) partners, a more integrated national surveillance system with improved electronic links among partners and improved surveillance systems for First Nations, Métis and Inuit communities. This article summarizes the recently updated Surveillance Annex of the CPIP (2).

\section{Objectives}

In support of the broader CPIP goals of minimizing serious illness and overall deaths and societal disruption, the objectives of the surveillance strategy are to provide timely and high-quality information to:

- Determine when and where influenza activity is occurring and who is being affected

- Determine and monitor underlying risk conditions associated with severe disease

- Describe clinical patterns of disease

- Assess and monitor the relative impact of the pandemic

- Detect changes in the antigenic and genetic character of the pandemic virus and its susceptibility to antiviral medications

- Support the implementation of interventions and the evaluation of their impact. 


\section{Canadian context}

Pandemic surveillance involves both epidemiologic and laboratory components and is built on the FPT surveillance systems that are already in place for seasonal influenza, taking advantage of existing and practised processes and linkages among jurisdictional and international public health entities. Seasonal surveillance systems include FluWatch, Canada's national influenza surveillance system, as well as the Immunization Monitoring Program ACTive (IMPACT), the Serious Outcomes Surveillance (SOS) Network and the Sentinel Practitioners Surveillance Network (SPSN). However, as seasonal surveillance systems do not provide data on the full spectrum of disease, pandemic surveillance can be augmented by special studies that focus on certain geographic regions, communities, or vulnerable groups within the population to obtain data on symptomatic individuals who do not seek health care and asymptomatically infected persons.

There are a number of uncertainties and variabilities associated with pandemic influenza that require specific surveillance capabilities and activities. As the timing and specific characteristics of a pandemic are not known in advance, pandemic surveillance must be scalable to different levels of impacts, and adaptable to changing conditions. Flexibility and adaptability are also necessary to respond appropriately to the variable conditions in different regions of Canada; due to Canada's size and the fact that pandemic conditions (e.g., intensity, timing and strain dominance) can differ by region. The geographic and sociocultural diversity in Canada's populations also requires flexibility to tailor surveillance activities to the needs and capacities of different regions and populations. Finally, surveillance activities during a pandemic must take into account ethical considerations, such as data confidentiality, to guard against unintentional stigmatization, and legal considerations, such as data-sharing agreements, to facilitate reporting requirements.

\section{Key elements in the surveillance strategy}

\section{Early detection and investigation}

Early detection and investigation of a novel influenza virus may occur through detection of signal events, such as cases or clusters of severe acute respiratory infections (SARI) and laboratory detections of novel influenza viruses; therefore, participation of hospitals in SARI surveillance is important for detecting novel viruses. When a novel virus is confirmed by laboratory testing and virus subtyping, local public health authorities will conduct case and contact investigations, with FPT support as required. In turn, PTs should report cases to PHAC within 24 hours to enable reporting to the World Health Organization (WHO) as required by the International Health Regulations (4).

\section{Community-based surveillance}

Community-based pandemic surveillance provides information on the occurrence of influenza illness, including data on clinical severity, age groups affected and risk factors associated with severe disease. This surveillance also provides data on the progress of the pandemic, enabling local authorities to plan response needs. Community-based surveillance is based on the seasonal FluWatch surveillance system, consisting of regular PT reporting of influenza activity levels and outbreaks of influenza-like-illnesses (ILI) to monitor geographic spread and trends over time, as well as syndromic surveillance, such as patient consultations for ILI, calls to PT telehealth systems and data on antiviral prescriptions and sales of over-the-counter medications relevant to influenza and ILI.

\section{Severe outcomes surveillance}

Severe outcomes surveillance (SOS), which captures data on severe outcomes, such as hospitalizations, intensive care unit admissions and deaths forms, is an important component of pandemic influenza surveillance. Data from SOS helps quantify the impact upon the health care system, identify high-risk conditions for prioritization of vaccines and antiviral recommendations, assess the effectiveness of the vaccine, and determine the need for additional public health measures. Seasonal SOS is provided through reports of hospitalizations and deaths from some PTs, as well as from IMPACT, a pediatric hospital-based surveillance network, and the SOS Network, a sentinel influenza network of hospitals that reports detailed case-based information on adult hospitalizations and deaths.

\section{Laboratory surveillance}

Laboratory surveillance includes routine weekly reports of respiratory virus detections, including the number of positive tests for influenza by type and subtype. These data are reported to FluWatch through the sentinel-laboratory-based Respiratory Virus Detection Surveillance System (RVDSS) (5) and also to the Global Influenza Surveillance and Response System through the WHO's FluNet (6). Public Health Laboratories (PHLs) also follow a protocol to submit a proportion of virus samples and patient specimens, which the National Microbiology Laboratory (NML) tests for strain characterization and antiviral resistance, to inform the ongoing immunization program or antiviral strategy during a pandemic. Guidance on the conduct of these laboratory functions by federal, provincial and front-line laboratories is provided in the Laboratory Annex (7) and is also summarized elsewhere in this issue of the Canada Communicable Disease Report (CCDR) (8).

\section{Special studies}

Routine seasonal influenza surveillance may not provide all the information that authorities need in a pandemic to understand the novel virus and determine the most appropriate interventions. Special studies may be required to gather information on community transmission and rates of infection and illness among specific populations. Planning for these studies needs to be in place in advance to enable rapid implementation in a pandemic. The Public Health Agency of Canada (PHAC) is a participant in the Consortium for Standardization of Influenza Seroepidemiology (CONSISE), an international initiative to develop a standardized approach to influenza studies and comprehensive influenza investigation protocols for pandemic studies (9). 


\section{Modelling}

Mathematical modelling, coordinated by PHAC in partnership with academics and public health agencies, can help support pandemic decision-making by helping predict the anticipated impact of a pandemic, the interventions that might be effective, and whether subsequent waves of disease may occur.

There are challenges in the use of surveillance data for modelling, including data quality and national representativeness. These challenges should be addressed during the interpandemic period by strengthening linkages between public health and modellers, developing data-sharing protocols, and establishing data standards and reporting requirements for modelling.

\section{Data collection, reporting and analysis}

Data collection and reporting rely on information generated by a number of sources. The PHAC receives and analyzes surveillance data collected by the PTs and from the NML, and reports key information back to PTs and internationally to the WHO. All epidemiological and clinical data need to be analyzed in a timely manner to assess the characteristics and impact of the pandemic. To enable these analyses during a pandemic, key epidemiological and clinical parameters should be characterized in advance.

The $2009 \mathrm{H} 1 \mathrm{~N} 1$ pandemic identified the need to improve the consistency of information captured in national influenza surveillance, as well as the need for formal FPT data-sharing agreements, electronic linkages to facilitate timely transfer of surveillance data, and sufficient human resources for data analysis and interpretation. There are ongoing FPT efforts to strengthen national influenza surveillance to address these issues.

\section{Integration with other response components}

Many surveillance activities are conducted in interaction with other components of the influenza response. These other components include the laboratory response, which is described in the Laboratory Annex (7) and in the summary of the laboratory strategy in this issue of CCDR (8).

Recommendations on the use of vaccines and on vaccine prioritization require epidemiological information and analysis of risk factors for severe disease. These activities and others, including monitoring of influenza strain and vaccine effectiveness, are detailed in the Vaccine Annex $(10,11)$. Surveillance data also support decisions on other interventions, such as the use of antivirals, and decisions on public health measures are based on epidemiological characteristics, while clinical care is influenced by information produced by early assessments of the impact of the pandemic.

Wild birds are the natural reservoir for the influenza virus. As such, surveillance on wildlife, poultry and other livestock is important to better understand influenza virus evolution and to assess pandemic threats. Formal linkages between public health and animal health authorities at the federal and PT levels are needed to strengthen surveillance activities and information sharing.

Another critical component of pandemic influenza response that relies on information provided by surveillance activities is communication with the public and with health care providers. A risk communication plan should be developed, on the basis of information produced by risk assessments; detailed guidance on this activity is provided in the Communications Annex (12).

\section{Research}

Prior to a pandemic, it is important to develop data standards and minimum data reporting requirements to facilitate the generation of consistent, high-quality data for epidemiological and modelling research conducted during a pandemic. In addition, it is important to undertake pre-planning activities, such as developing detailed protocols with pre-approval by the appropriate regulatory and research ethics boards to allow quick implementation of research projects during a pandemic.

\section{Assessment and evaluation}

Routine seasonal influenza surveillance offers an opportunity for practice and for piloting and evaluating new surveillance strategies. In addition, after a pandemic, surveillance programs should be evaluated in each jurisdiction and comparisons made to identify lessons learned and best practices.

\section{Discussion}

A major principle underpinning the surveillance strategy is the value of using existing Canadian structures and networks in place for seasonal influenza as the basis for pandemic surveillance activities. Improvements in the surveillance system are still needed, however, including consistency of information capture, FPT data-sharing agreements, and electronic links for transferring data. As much of this work as possible should be done in advance. Work to standardize data collection and improve data transfer should be conducted in the interpandemic period, and where possible integrated into the seasonal influenza surveillance system, which will enhance pandemic surveillance capabilities. Consistency in reporting can be improved through the development of standard reporting templates and timelines to be used by PTs and PHAC; these will be enabled by improvements in infrastructure such as electronic databases, immunization registries in all jurisdictions, and secure electronic or web-based reporting mechanisms.

Seasonal influenza surveillance is conducted every year and provides an opportunity for an evaluation of existing strategies and arrangements, and to trial new activities. Periodic outbreaks are additional opportunities for the testing of coordinated and rapid response, including rapid deployment and reporting of research studies. Guidelines and indicators for evaluating surveillance systems have been produced by the WHO (13) and the US Centers for Disease Control and Prevention (CDC) (14) and are available for use to assist with this activity. 


\section{Conclusion}

The surveillance strategy guides FPT governments in developing their plans and ensuring their capacity to fulfill their roles and collaborate effectively with other jurisdictions in an influenza pandemic. As with other components of the CPIP, it is an evergreen strategy, and the state of preparedness of the surveillance system is subject to ongoing evaluation, with improvements and updates incorporated as appropriate.

\section{Authors' statement}

Canadian Pandemic Influenza Preparedness Task Group (CPIPTG) Members: Henry B (Chair), Alfieri A, Gant S, Gemmill I, Hatchette T, Jayaraman G, Schwartz B

CPIPTG Secretariat: Paddle L, Stirling R, Gadient S

PHAC: Charos G, Williams J

\section{Conflict of interest}

None.

\section{Acknowledgements}

Many thanks to Anne Wiles who prepared the initial draft of this summary.

\section{Funding}

The work of the Canadian Pandemic Influenza Preparedness Task Group is supported by the Public Health Agency of Canada.

\section{References}

1. World Health Organization. Public Health Surveillance. http://www.who.int/topics/public_health_surveillance/en/

2. Public Health Agency of Canada. Canadian Pandemic Influenza Preparedness: Planning Guidance for the Health Sector, Surveillance Annex. Ottawa (ON): PHAC; 2015.https://www.canada.ca/en/public-health/services/ flu-influenza/canadian-pandemic-influenza-preparedness-p lanning-guidance-health-sector/surveillance-annex.html

3. Public Health Agency of Canada. Canadian pandemic influenza preparedness: Planning guidance for the health sector Ottawa (ON): PHAC; 2015. http://www.phac-aspc. gc.ca/cpip-pclcpi/

4. Health Organization. 2005, International Health Regulations Third Edition. http://apps.who.int/iris/bitstream/10665/2461 07/1/9789241580496-eng.pdf?ua=1
5. Public Health Agency of Canada. Respiratory Virus Detections in Canada. Otttawa (ON); PHAC; 2017. https:// www.canada.ca/en/public-health/services/surveillance/ respiratory-virus-detections-canada.html

6. World Health Organization FLuNet. http://www.who.int/ influenza/gisrs_laboratory/flunet/en/

7. Public Health Agency of Canada. Canadian pandemic influenza preparedness: Planning guidance for the health sector. Ottawa (ON): PHAC; 2015. https://www.canada.ca/ en/public-health/services/flu-influenza/canadian-pandemi c-influenza-preparedness-planning-guidance-health-sector/ laboratory-annex.html

8. Henry B on behalf of Canadian Pandemic Influenza Preparedness Task Group. Canada's Pandemic Influenza Preparedness: laboratory strategy. Can Commun Dis Rep. 2018;44(1):10-3.https://www.canada.ca/en/ public-health/services/reports-publications/canad a-communicable-disease-report-ccdr/monthly-issue/2018-44/ ccdr-volume-44-1-january-4-2018/canadas-pandemi c-laboratory-strategy.html

9. Consortium for the Standardization of Influenza Seroepidemiology. CONSISE; 2017. https://consise.tghn.org/

10. Public Health Agency of Canada. Vaccine annex: Canadian pandemic influenza preparedness: Planning guidance for the health sector. Ottawa (ON): PHAC; 2017. https:// www.canada.ca/en/public-health/services/flu-influenza/ canadian-pandemic-influenza-preparedness-planning-gu idance-health-sector/vaccine-annex.html

11. Henry B. Gadient S on behalf of Canadian Pandemic Influenza Preparedness Task Group. Canada's pandemic vaccine strategy. Can Commun Dis Rep. 2017;43(7/8):160-3. https://www.canada.ca/content/dam/phac-aspc/migration/ phac-aspc/publicat/ccdr-rmtc/17vol43/dr-rm43-7-8/assets/ pdf/17vol43_7_8-ar-05-eng.pdf

12. Public Health Agency of Canada. Canadian pandemic Influenza plan for the health sector. Ottawa (ON): PHAC, 2009. https://www.canada.ca/content/dam/phac-aspc/ migration/phac-aspc/cpip-pclcpi/assets/pdf/annex_k-eng. pdf. [Communications Annex].

13. World Health Organization. WHO interim global. Epidemiological surveillance standards for influenza. http:// www.who.int/influenza/resources/documents/WHO_ Epidemiological_Influenza_Surveillance_Standards_2014.pdf

14. German RR, Lee LM, Horan JM, Milstein RL, Pertowski CA, Waller MN. Guidelines Working Group Centers for Disease Control and Prevention (CDC). Updated guidelines for evaluating public health surveillance systems: recommendations from the Guidelines Working Group. MMWR Recomm Rep 2001 Jul;50 RR-13:1-35. PubMed (https://www.ncbi.nlm.nih.gov/entrez/query.fcgi?cmd=Retriev e\&db=PubMed\&list_uids=18634202\&dopt=Abstract). 